\title{
LEMME DE MOSER FEUILLETÉ ET CLASSIFICATION DES VARIÉTÉS DE POISSON RÉGULIÈRES
}

\author{
G. HECTOR, E. MACIAS ET M. SARALEGI
}

\begin{abstract}
Regular Poisson structures with fixed characteristic foliation $\mathcal{F}$ are described by means of foliated symplectic forms. Associated to each of these structures, there is a class in the second group of foliated cohomology $H^{2}(\mathcal{F})$. Using a foliated version of Moser's lemma, we study the isotopy classes of these structures in relation with their cohomology class. Explicit examples, with $\operatorname{dim} \mathcal{F}=2$, are described.
\end{abstract}

\section{Cohomologie feuilletée et variétés de Poisson régulières}

Soit $(M, \mathcal{F})$ une variété munie d'un feuilletage régulier $\mathcal{F}$. On introduit le complexe des formes feuilletées puis la notion de forme feuilletée symplectique qui servira à décrire les variétés de Poisson régulières.

\subsection{Formes feuilletées-cohomologie feuilletée.}

Soient $\left(\Omega^{*}(M), d\right)$ le complexe de DeRham de $M$ et $\Omega^{*}(M, \mathcal{F})$ le sous-complexe des formes relatives défini par $\omega \in \Omega^{r}(M, \mathcal{F})$ si $\omega \in \Omega^{r}(M)$ et

$$
\omega\left(X_{1}, X_{2}, \ldots, X_{r}\right)=0
$$

pour tout $r$-uple de champs tangents à $\mathcal{F}$. Le complexe $\Omega^{*}(\mathcal{F})$ des formes $\mathcal{F}$-feuilletées est défini par passage au quotient:

$$
\Omega^{*}(\mathcal{F})=\Omega^{*}(M) / \Omega^{*}(M, \mathcal{F})
$$

et sa cohomologie $H^{*}(\mathcal{F})$ est la cohomologie feuilletée de $(M, \mathcal{F})$. Pour $r>\operatorname{dim} \mathcal{F}$, on a $\Omega^{r}(\mathcal{F})=0$ et donc $H^{r}(\mathcal{F})=0$ mais même si $M$ est compacte on ne peut en général rien conclure pour $H^{n}(\mathcal{F}), n=\operatorname{dim} \mathcal{F}$.

Concernant la structure de $\Omega^{*}(\mathcal{F})$, on remarquera que:

i) $\Omega^{*}(M, \mathcal{F})$ étant un idéal de $\Omega^{*}(M)$, le produit extérjeur $\wedge$ définit par passage au quotient une structure multiplicative sur $\Omega^{*}(\mathcal{F})$; 
ii) le produit intèrieur $X\rfloor \alpha$ de $\alpha \in \Omega^{*}(\mathcal{F})$ avec un champ $X$ tangent à $\mathcal{F}$ est un élément bien défini de $\Omega^{*-1}(\mathcal{F})$; on pourra donc définir la dérivée de Lie $L_{X} \propto$ par la formule habituelle; elle s'annulera exactement si $\alpha$ est invariante par le flot engendré par $X$;

iii) de mêrme, l'évaluation de $\alpha \in \Omega^{r}(\mathcal{F})$ sur un $r$-champ tangent à $\mathcal{F}$ est un èlément de $\Omega^{0}(\mathcal{F})=\Omega^{0}(M)$ donc une function sur $M$; en particulier on pourra définir comme d'habitude le rang de $\alpha$ en un point.

\subsection{Formes feuilletées symplectiques et variétés de Poisson.}

Suppons que $\mathcal{F}$ est de dimension paire $2 \mathrm{~m}$. On dira qu'une forme $\sigma \in \Omega^{2}(\mathcal{F})$ est une forme feuilletée symplectiquse si elle est fermée et de rang $2 m$. Cette dernière condition est équivalente au fait que $\stackrel{m}{\wedge} \sigma$ est non nulle en tout point i.e. $\stackrel{m}{\wedge} \sigma$ est une forme feuilletée volume.

Le couple $(\mathcal{F}, \sigma)$ définit une structure de Poisson $\Lambda$ sur $M$ dont le feuilletage caractéristique est égal à $\mathcal{F}$. En effet, parce que $\sigma$ est de rang $2 m$, il existe pour tout $f \in \Omega^{0}(M)$, un unique champ de vecteurs $X_{f}$ tangent à $\mathcal{F}$ tel que:

$$
\left.X_{f}\right\rfloor \sigma=-\overline{d f}
$$

(où $\overline{d f}$ est la classe dans $\Omega^{1}(\mathcal{F})$ de $d f \in \Omega^{\mathbb{1}}(M)$ ); et le bivecteur $\Lambda$ défini par

$$
\Lambda(d f, d g)=\sigma\left(X_{f}, X_{g}\right)
$$

est une structure de Poisson dont les Hamiltoniens sont les champs $X_{f}$ qui engendrent évidemment $\mathcal{F}$.

Réciproquement, si $\Lambda$ est une structure de Poisson régulière de rang constant $2 m$ sur une variété $M$ de dimension $(2 m+n)$, il existe un systeme de cartes locales

$$
\left(V, x^{1}, \ldots, x^{m}, y^{1}, \ldots, y^{m}, z^{1}, \ldots, z^{n}\right)
$$

telles que:

i) le feuilletage caractéristique $\mathcal{F}$ de $\Lambda$ est défini par les équations $d z^{j}=0$;

ii) en restriction à $V$, la forme symplectique sur les feuilles de $\mathcal{F}$ s'écrit $\omega_{\mathcal{F}}=\sum_{i=1}^{m} d x^{i} \wedge d y^{i}$. (cf. $[\mathrm{L}]$ ou $[\mathbf{W}]$ ). Le choix d'un supplémentaire de $T(\mathcal{F})$ permet d'étendre la famille $\left(\omega_{\mathcal{F}}\right)$ en une 2 -forme sur $M$ dont la classc $\sigma$ dans $\Omega^{2}(\mathcal{F})$ est une forme feuilletée symplectique independante du choix du supplémentaire.

En résumé, toute structure de Poisson, régulière $\Lambda$ est exactement déterminée par la donnée d'un couple $(\mathcal{F}, \sigma)$ où $\mathcal{F}$ est un feuilletage régulier et $\sigma$ est une 2 -forme $\mathcal{F}$-feuilletée symplectique. Pour $\mathcal{F}$ fixé, on dira que $\Lambda=(\mathcal{F}, \sigma)$ est supportée par $\mathcal{F}$ et on désignera par Poiss $(\mathcal{F})$ l'espace des structures de Poisson de support $\mathcal{F}$. 


\subsection{Classe de cohomologie associée à $\Lambda=(\mathcal{F}, \sigma)$.}

A toute structure de Poisson $\Lambda=(\mathcal{F}, \sigma)$ on associe la classe de cohomologie feuilletée

$$
[\Lambda]=[\sigma] \in H^{2}(\mathcal{F})
$$

et on dit que

i) $\Lambda$ est présymplectique si $[\Lambda] \in \operatorname{Im}\left\{H^{2}(M) \rightarrow H^{2}(\mathcal{F})\right\}$ i.e. $\sigma$ admet un représentant $\omega \in \Omega^{2}(M)$ qui est une forme fermée;

ii) $\Lambda$ est exacte si $[\Lambda]=0$ i.e. $\sigma$ admet un represéntant $\omega$ qui est exact.

La classe $[\Lambda]$ joue pour les structures de Poisson de support $\mathcal{F}$ le rôle joué par la classe de la forme symplectique pour les variétés symplectiques.

\section{Lemme de Moser feuilleté}

Classifier les structures de Poisson régulières de support $(M, \mathcal{F})$ fixé, va consister à étudier l'application

$$
\begin{array}{clc}
\chi: \operatorname{Poiss}(\mathcal{F}) & \longrightarrow & H^{2}(M) \\
\Lambda & \longrightarrow & {[\Lambda]}
\end{array}
$$

Pour ce faire, on se servira d'une version feuilletée du lemme de Moser (cf. $[\mathrm{M}]$ ).

2.1. Comparaison de structures $\Lambda=(\mathcal{F}, \sigma)$.

i) Une famille à un paramètre de formes feuilletées étant définie par passage au quotient d'une famille à un paramètre de formes differentielles sur $M$, on dira que $\Lambda_{t}=\left(\mathcal{F}, \sigma_{t}\right), t \in \mathbf{R}$, est une famille à un paramètre de structures de Poisson cohomologues, s'il existe une famille à un paramètre $\lambda_{t} \in \Omega^{1}(\mathcal{F})$ telle que

$$
\sigma_{t}=\sigma_{0}+d \lambda_{t}
$$

ii) Par ailleurs, soit $\varphi: \mathbf{R} \times M \rightarrow M$ un fot sur $M$ engendré par un champ de vecteurs $X$ tangent à $\mathcal{F}$. Alors, $\varphi_{t}$ agit sur $\Omega^{*}(\mathcal{F})$ et si $\sigma \in \Omega^{2}(\mathcal{F})$ est symplectique, il en sera de même pour $\varphi_{t}^{*} \sigma$. On dira que $\varphi_{t}^{*} \sigma$ est une famille de structures de Poisson isotopes.

Puur $t$ fxé, on vérifie immediatement que l'opérateur d'homotopie asocié à l'isotopie $\left(\varphi_{b i}\right)_{b \in[0,1]}$ passe au quotient en un opérateur d'homotopie

$$
H_{t}: \Omega^{*}(\mathcal{F}) \longrightarrow \Omega^{*-1}(\mathcal{F})
$$

qui vérifie:

$$
\varphi_{t}^{*}-\mathrm{id}^{*}=d H_{t}+H_{t} d .
$$


Donc $\varphi_{i}^{*} \sigma-\sigma=d H_{i} \sigma$ et $\varphi_{t}^{*} \sigma$ est une familie à un paramètre de structures cohomologues.

On se propose maintenant de vérifier le réciproque dans le cas oì $M$ est compacte. Pour cela soient $p$ la projection de $\mathbf{R} \times M$ sur $M$ et $\hat{\mathcal{F}}=p^{*} \mathcal{F}$. Si $\alpha_{t} \in \Omega^{r}(\mathcal{F})$ est une famille à un paramètre de formes $\mathcal{F}$-fetuilletées, on désigne par

$\hat{\alpha_{t}} \in \Omega^{r}(\hat{\mathcal{F}})$ la $r$-forme $\hat{\mathcal{F}}$-feuilletée, de type $(0, r)$ relativement à la décomposition naturelle de $T(\mathbf{R} \times M)$ et égale à $\alpha_{t}$ en restriction à $\{t\} \times M$;

$\alpha_{t}^{\prime}$ la famille à un paramètre de $r$-formes $\mathcal{F}$-feuilletées obtenue en dérivant par rapport à $t$ les coefficients de $\alpha_{\ell}$.

On a les relations:

$$
(2.1 .1)\left\{\begin{array}{l}
\left(d \alpha_{t}\right)^{\prime}=d\left(\alpha_{t}^{\prime}\right) \text { et }\left(\alpha_{t}\right)^{t}=\widehat{\alpha_{t}^{\prime}} \\
d \hat{\alpha}_{t}=\widehat{d \alpha_{t}}+d t \wedge \hat{\alpha}_{t}^{\prime} .
\end{array}\right.
$$

De même si $Y_{z}$ est une farnille à un paramètre de champs de vecteurs sur $M$, on désignera par $\hat{Y}_{t}$ le champ de vertical sur $\mathrm{R} \times M$ dont la restriction à $\{t\} \times M$ est donnée par $Y_{t}$.

2.2. Lemme. Supposons $M$ compacte. Pour toute famille $\grave{a}$ un paramètre $\sigma_{t}=\sigma_{0}+d \lambda_{t}$ de structures de Poisson cohomologues, il existe un flot $\varphi$ tangent à $\mathcal{F}$ tel que

$$
\sigma_{t}=\varphi_{t}^{*} \sigma_{0}
$$

Démonstration: D'après (2.1.1), la 2-forme $\hat{\mathcal{F}}$-feuilletée $\omega$ définie par

$$
\omega=\hat{\sigma}_{t}+d t \wedge \hat{\lambda}_{t}^{\prime}=\hat{\sigma}_{0}+\widehat{d \lambda_{t}}+d t \wedge \hat{\lambda}_{t}^{\prime}
$$

s'écrit encore $\omega=\hat{\sigma}_{0}+d \dot{\lambda}_{1}$; elle est donc fermée.

En outre, si $X_{t}$ est le champ de vecteurs tangent à $\mathcal{F}$ défini par l'équation

$$
\left.X_{t}\right] \sigma_{t}=-\lambda_{t}^{\prime},
$$

on aura $\left.X_{t}\right\rfloor \lambda_{t}^{\prime}=0$ et donc

$$
\left.\left.\hat{X}_{t}\right\rfloor \omega=\hat{X}_{t}\right\rfloor \hat{\sigma}_{t}=-\hat{\lambda}_{t}^{t} .
$$

Par suite si $z=\frac{\partial}{\partial t}+\hat{X}_{t}$, il vient:

$$
Z\rfloor \omega=0 .
$$

On en déduit que la dérivée de Lie $L_{Z} \omega$ est nulle; le flot $\hat{\varphi}_{\iota}$ engendré par $Z$ préserve $\hat{\mathcal{F}}$ et $\omega$ et donc le fiot $\varphi_{t}$ sur $M$ obtenu par projection de $\hat{\varphi}_{t}$ sur $M=\{0\} \times M$ est tel que $\varphi_{t}^{*} \sigma_{0}=\sigma_{t}$

En résumé, on obtient le lemme de Moser feailleté: 
2.3. Théorème. Pour les structures de Poisson supportées par un feuilletage $\mathcal{F}$ sur une variété compacte $M$, les deux conditions suivantes sont équivalentes:

i) $\sigma_{t}$ est une famille $\dot{a}$ un paramètre de structures cohomologues;

ii) Il existe une isotopie $\varphi$ tangente à $\mathcal{F}$ telle que $\sigma_{t}=\varphi_{t}^{*} \sigma_{0}$.

$\mathrm{Si} \mathcal{F}$ est de dimension 2, une forme feuilletée symplectique est aussi une forme feujlletée volume qui défnit une orientation de $\mathcal{F}$ et on trouve un résultat plus précis:

2.4. Corollaire. Si $\operatorname{dim} \mathcal{F}=2$, deux structures de Poisson $\Lambda_{i}=\left(\mathcal{F}, \sigma_{i}\right)$, $i=0,1$, sont isotopes si et seulement si $\sigma_{0}$ et $\sigma_{1}$ définissent la même orientation de $\mathcal{F}$ et

$$
\left[\sigma_{0}\right]=\left[\sigma_{1}\right] \in H^{2}(\mathcal{F})
$$

Démonstration: En effet, si $\sigma_{0}$ et $\sigma_{1}$ définissent la mëme orientation de $\mathcal{F}$ et sont cohomologues dans $H^{2}(\mathcal{F})$, il existe une fonction $g>0$ et $\lambda \in \Omega^{1}(\mathcal{F})$ tels que $\sigma_{1}=g \sigma_{0}$ et $\sigma_{1}-\sigma_{0}=d \lambda$. Pour tout $t \in[0,1]$,

$$
\sigma_{t}=t \sigma_{1}+(1-t) \sigma_{0}=(t g+1-t) \sigma_{0}
$$

est une forme feuilletée symplectique et

$$
\sigma_{t}-\sigma_{0}=t \sigma_{1}-t \sigma_{0}=t d \lambda .
$$

Donc $\Lambda_{t}=\left(\mathcal{F}, \sigma_{t}\right)$ est une famille à un paramètre de structures de Poisson cohomologues. On applique 2.3 . (en se restreignant à l'intervalle $[0,1]$ )

\subsection{Application: le cône $\mathcal{J}$ Poiss $(\mathcal{F})$.}

Si $\operatorname{dim} \mathcal{F}=2$, toute forme feuilletée volume est une forme feuilletée symplectique et donc Poiss $(\mathcal{F}) \neq \phi$. En outre, d'après (2.3.), l'application $\chi$ identifie l'ensemble des classes d'isotopie de structures de Poisson avec l'image de $\chi$ qui est un cône ouvert de $H^{2}(\mathcal{F})$ que l'on désignere par $\mathcal{J}$ Poiss $(\mathcal{F})$. Enfin dans les exemples du $\S 3$, on décrira plus precisément le cône $\mathcal{J}$ Poiss ${ }^{+}(\mathcal{F})$ des classes d'isotopie de structures de Poisson $\Lambda=(\mathcal{F}, \sigma)$ positives i.e. telles que l'orientation définie par $\sigma$ coincide avec une orientation préalablement fixée de $\mathcal{F}$.

\section{Exemples}

Pour finir, on va donc décrire le cône $\mathcal{J}$ Poiss ${ }^{+}(\mathcal{F})$ des classes d'isotopie de structures de Poisson positives supportées par certains feuilletages $\mathcal{F}$ de dimension 2 sur une variété $M$. 


\subsection{Fibrations localement triviales à fibres compactes.}

Supposons $\mathcal{F}$ défini par une fibration localement triviale $\pi: M \rightarrow B$ à fibres compactes de dimension 2. L'integration sur les fibres de $\pi$ est une application linéaire surjective de $\Omega^{2}(M)$ sur $C^{\infty}(B)$ nulle sur $\Omega^{2}(M, \mathcal{F})$ donc induit un opérateur surjectif

$$
f: \Omega^{2}(\mathcal{F}) \longrightarrow C^{\infty}(B)
$$

dont le noyau est exactement $d \Omega^{1}(\mathcal{F})$. Il induit un isomorphisme

$$
f^{*}: H^{2}(\mathcal{F}) \longrightarrow C^{\infty}(B)
$$

qui identifie $\mathcal{J}$ Poiss ${ }^{+}(\mathcal{F})$ avec le cône $C_{+}^{\infty}(B)$ des fonctions strictement positives sur $B$ (la compacite de $M$ dans 2.3 . est remplacée ici par la compacité des fibres!)

3.2. Feuilletages Iinéaires de $T^{3}$.

Soit $\mathcal{F}_{w}$ le feuilletage de $T^{3}$ défini par la 1 -forme fermée, à coefficients constants, $\omega=\alpha d x+\beta d y+d z$ sur $T^{3}=\mathbb{R}^{3} / \mathbb{Z}^{3}$ et soit $r$ le rang sur $Q$ du triplet $(\alpha, \beta, 1)$.

i) Si $r=1, \mathcal{F}_{w}$ est un fibré trivial de fibre $T^{2}$ et base $S^{1}$ et d'après (3.1.), $\mathcal{J}$ Poiss ${ }^{+}\left(\mathcal{F}_{\omega}\right)$ est un cône de dimension infinie;

ii) si $r=2$, toutes les feuilles de $\mathcal{F}_{w}$ sont des cylindres partout denses dans $T^{3}$ et il existe une fibration triviale,

$$
\$^{3} \longrightarrow T^{3} \stackrel{\bar{u}}{\longrightarrow} T^{2}
$$

telle que $\omega=\bar{u}^{*} \eta$ où $\eta$ est une 1 -forme à coefficents constants sur $T^{2}$ du type. Par integration sur les fibres de $\bar{u}$, on montre que $H^{2}\left(\mathcal{F}_{\omega}\right) \cong$ $H^{1}\left(\mathcal{F}_{\eta}\right)$ et ce dernier groupe est de dimension 1 ou infinie suivant que $\alpha$ vérifie ou non une condition diophantienne. Donc $\mathcal{J}$ Poiss ${ }^{+}\left(\mathcal{F}_{\omega}\right)$ sera suivant le cas de dimension 1 ou infinie.

iii) le cas $r=3$ sera analogue à $r=2$ sauf que cette fois-ci les feuilles de $\mathcal{F}_{w}$ des plans et non plus des cylindres.

On remarquera que dans tous les cas, $\mathcal{F}_{w}$ n'admet pas de structure de Poisson exacte car si la forme feuilletée symplectique $\sigma$ était représentée par $d \mu \in \Omega^{2}\left(T^{3}\right)$, alors $\sigma \wedge \omega=d(\mu \wedge \omega)$ serait une forme volume exacte sur la variété compacte $T^{3}$.

3.3. Un exemple de structure de Poisson exacte sur une variété compacte.

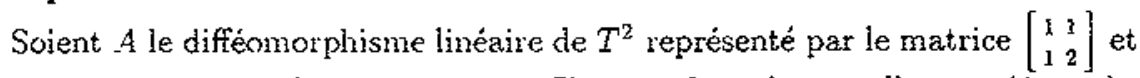
$\lambda$ une valeur propre de cette matrice. Si $\eta=\alpha d x+d y$ est telle que $A^{*} \eta=\lambda \eta$ alors $\alpha$ est algébrique donc vérifie une condition disphantienne et le feuilletage $\mathcal{F}_{\eta}$ défini par $\eta$ est tel que $H^{1}\left(\mathcal{F}_{\eta}\right)=\mathrm{R}$. 
Soit $T_{A}^{3}$ l'espace total du fibré en tores au-dessus de $\$^{1}$ de monodromie $A$; c'est le quotient de $\mathbf{R} \times T^{2}$ par l'action de $\mathbb{Z}$ engendrée par le difféomorphisme $\tilde{A}(t, u)=(t+1, A(u))$. On le munit du feuilletage $\mathcal{F}_{A}$ obtenu par passage au quotient du feuilletage $\tilde{\mathcal{F}}_{\eta}=p r_{2}^{*} \mathcal{F}_{\eta}$. En procédant de façon tout à fait analogue à la situation classique, on construit une suite exacte de Wang en cohomologie feuilletée qui s'écrit:

$$
\longrightarrow H^{1}\left(\mathcal{F}_{\eta}\right) \stackrel{A^{*}-I}{\longrightarrow} H^{1}\left(\mathcal{F}_{\eta}\right) \longrightarrow H^{2}\left(\mathcal{F}_{A}\right) \rightarrow H^{2}\left(\mathcal{F}_{\eta}\right) \longrightarrow
$$

Le générateur de $H^{1}\left(\mathcal{F}_{\eta}\right)$ est vecteur propre pour la valeur propre irrationnelle $1 / \lambda$ donc $A^{*}-I \neq 0$ est un isomorphisme de $H^{1}\left(\mathcal{F}_{\eta}\right)$ sur lui-même et comme $H^{2}\left(\mathcal{F}_{\eta}\right)=0$, il vient $H^{2}\left(\mathcal{F}_{A}\right)=0$. Bref toute structure de Poisson supportée par $\mathcal{F}_{A}$ est exacte.

\subsection{Feuilletages avec cycles évanouissants.}

Soit $\mathcal{F}$ un feuilletage de codimension 1 sur une variété compacte de dimension 3. D'après le théorème de Novikov, on sait que l'existence d'un cycle évanouissant non trivial est équivalente à l'existence d'une composante de Reeb (cf. $[\mathrm{HH}]$ ). Or on montre dans $[\mathrm{H} . \mathrm{LV}]$ que le second groupe de cohomologie feuilletée d'une telle composante est de dimension infinie; il en est donc de même pour tout fetilletage $\mathcal{F}$ admettant un cycle évanouissant non trivial.

Comme conséquence $\mathcal{J}$ Poiss ${ }^{+}(\mathcal{F})$ est de dimension infinie pour un tel $\mathcal{F}$.

\section{References}

[HH] G. HECTOR AND U. HIRSCH, Introduction to the Geometry of foliations, $A$ and $B$, Vieweg, (1981-83).

[H. LV] G. Hector et C. LASSO DE LA Vega, Structures de Poisson régulières et cycles évanouissants, (à paraitre).

[L] A. LICHNÉRowiCZ, Les variétés de Poisson et leurs algèbres de Lie associées, J. Diff. Geom. 12 (1977), 253-300.

[M] J. MOSER, On the volume elements on a manifold, Trans. Amer, math. Soc. 120 (1965), 286-294.

[W] A. WeISntein, The local structure of Poisson manifolds, J. Diff. Geom. $18(1983), 523-557$. 
G. Hector: URA CNRS 746

Institut de Mathemàtiques et Informatique

43 B. du 11 Novembre 1918

69622-Villeurbann-Cedex

FRANCE

E. Macias: Facultad de Matemáticas

Universidad Santiago de Compostela

SPAIN

M. Saralegi: C/ Chantada 21, Io .1a.

28029-Madrid

SPAIN

Rebut el 26 de Juliol de 1989 